\title{
CONSTRUCTING MULTIDIMENSIONAL PERIODIC CONTINUED FRACTIONS IN THE SENSE OF KLEIN
}

\author{
O. N. KARPENKOV
}

\begin{abstract}
We consider the geometric generalization of ordinary continued fractions to the multidimensional case introduced by F. Klein in 1895. A multidimensional periodic continued fraction is the union of sails with some special group acting freely on these sails. This group transposes the faces. In this article, we present a method of constructing "approximate" fundamental domains of algebraic multidimensional continued fractions and an algorithm testing whether this domain is indeed fundamental or not. We give some polynomial estimates on the number of the operations for the algorithm. In conclusion we present an example of a fundamental domain calculation for a two-dimensional series of two-dimensional periodic continued fractions.
\end{abstract}

\section{INTRODUCTION, DEFINITIONS, AND BACKGROUND}

The main goal of this paper is to introduce a new method for constructing the fundamental domains of multidimensional periodic continued fractions in the sense of Klein. The problem of generalizing ordinary continued fractions to the higher-dimensional case was posed by C. Hermite 9 in 1839. A large number of attempts to solve this problem led to the birth of several different remarkable theories of multidimensional continued fractions. In this paper we consider the geometrical generalization of ordinary continued fractions to the multidimensional case introduced by F. Klein in 1895 and published by him in [15] and [16.

A number of properties for ordinary continued fractions possesses multidimensional analogies. H. Tsuchihashi 38] found the connection between periodic multidimensional continued fractions and multidimensional cusp singularities. J.-O. Moussafir in [27] and O. German in [7] described a relationship between sails of multidimensional continued fractions and Hilbert bases. M. L. Kontsevich and Yu. M. Suhov discussed the statistical properties of the boundary of a random continued fraction in [17. The papers [35] and [36] by B. F. Skubenko and 8 by O. N. German are dedicated to the generalization of ordinary continued fractions with bounded integer edge lengths (the numbers that correspond to such continued fractions are the numbers with the worst possible rational approximations). For the classical theory of ordinary continued fractions we refer to the book 10 by A. Ya. Hinchin. V. I. Arnold in his article 2 and the book [1] proposed many questions on the geometry and combinatorics of continued fractions (including the questions on the study

Received by the editor May 12, 2005 and, in revised form, June 9, 2008.

2000 Mathematics Subject Classification. Primary 11J70; Secondary 11Y16.

Key words and phrases. Multidimensional continued fractions, convex polygons, integer lattices.

The author was supported by SS-1972.2003.1 and RFBR-05-01-01012a grants.

(C)2008 American Mathematical Society
Reverts to public domain 28 years from publication 1687 
of properties for such notions as affine types of the faces of the sails, their quantities and frequencies, integer angles between the faces, integer distances, volumes and so on).

Some examples of the periodic continued fractions were calculated in the papers [18, 20], and [21] by E. Korkina, 22] and [23] by G. Lachaud, [5], 30, 31, [32], and [33] by A. D. Bruno and V. I. Parusnikov, 11] and [12] by the author. A nice collection of two-dimensional continued fractions was presented by K. Briggs; see [4].

0.1. Definition of periodic multidimensional continued fractions. In this section we recall some basic notions and definitions (see also [13]). Consider a space $\mathbb{R}^{n+1}(n \geq 1)$ over $\mathbb{R}$. A point of $\mathbb{R}^{n+1}$ is said to be an integer point if all its coordinates are integers. Two sets are called integer-affine (integer-linearly) equivalent if there exists an affine (linear) transformation of $\mathbb{R}^{n+1}$ preserving the set of all integer points, and transforming the first set to the second. A plane is called an integer plane if it is integer-affine equivalent to some plane passing through the origin and containing the sublattice of the integer lattice, and the rank of the sublattice is equivalent to the dimension of the plane. A polyhedron is said to be an integer polyhedron if all its vertices are integers.

Consider an integer plane and an integer point in the complement to the plane. Let the Euclidean distance from the given point to the given plane equal $l$. The minimal value of nonzero Euclidean distances from integer points of the span of the given plane and the given point to the plane is denoted by $l_{0}$. The ratio $l / l_{0}$ is said to be the integer distance from the given integer point to the given integer plane.

Consider arbitrary $n+1$ hyperplanes in $\mathbb{R}^{n+1}$ that intersect at a unique point, namely the origin. The complement of the union of these hyperplanes consists of $2^{n+1}$ open orthants. Let us choose an arbitrary orthant.

Definition 0.1. The boundary of the convex hull of all integer points except the origin in the closure of the orthant is called the sail of the orthant. The set of all $2^{n+1}$ sails is called the $n$-dimensional continued fraction constructed according to the given $n+1$ hyperplanes.

Two $n$-dimensional continued fractions are said to be equivalent if the union of all sails of the first continued fraction is integer-linear equivalent to the union of all sails of the second continued fraction.

Definition 0.2. An operator in the group $S L(n+1, \mathbb{Z})$ is called an integer irreducible hyperbolic operator if the following conditions hold:

i) the characteristic polynomial of this operator is irreducible over $\mathbb{Q}$;

ii) all its eigenvalues are distinct and real.

Consider some integer irreducible hyperbolic operator $A \in S L(n+1, \mathbb{Z})$. Let us take the $n$-dimensional spaces that span all subsets of $n$ linearly independent eigenvectors of the operator $A$. The spans of every set of $n$ eigenvectors uniquely define $n+1$ hyperplanes passing through the origin in general position. These hyperplanes uniquely define the multidimensional continued fraction associated to $A$.

Definition 0.3. An n-dimensional continued fraction associated to some integer irreducible hyperbolic operator $A$ is called an $n$-dimensional continued fraction of 
an $(n+1)$-algebraic irrationality. The case of $n=1(2)$ corresponds to one(two)dimensional continued fractions of quadratic (cubic) irrationalities.

Now we formulate the notion of periodic continued fraction associated with an algebraic irrationality. Let $A$ be an integer irreducible hyperbolic operator. Denote by $\Xi(A)$ the set of all integer operators commuting with $A$. These operators form a ring with standard matrix addition and multiplication. (As a group $\Xi(A)$ is isomorphic to $\mathbb{Z}^{n+1}$.)

Consider the subset of the set $S L(n+1, \mathbb{Z}) \cap \Xi(A)$ that consists of all operators with positive real eigenvalues and denote it by $\bar{\Xi}(A)$. From the Dirichlet unit element theorem (see [3]) it follows that the subset $\bar{\Xi}(A)$ forms a multiplicative Abelian group isomorphic to $\mathbb{Z}^{n}$, and that its action is free. Any operator of this group preserves the integer lattice and the union of all $n+1$ hyperplanes, and hence it takes the $n$-dimensional continued fraction onto itself bijectively. (Whenever all eigenvalues are positive, the sails are also taken onto themselves in a one-to-one way.) In addition, the quotient of a sail under this group action is isomorphic to an $n$-dimensional torus. These statements are based on the generalization of the Lagrange theorem on ordinary continued fractions. The combinatorial topological generalization of Lagrange theorem was obtained by E. I. Korkina in [19] and its algebraic generalization by G. Lachaud 22 .

Theorem 0.4 (E. I. Korkina, 19]). Consider some orthant $C$ and the sail $K(C)$ corresponding to this orthant. If there exists a combinatorial isomorphism of the sail $V(C)$ that preserves the combinatorial structure of the sail, then there exists an operator in the group $G L(n+1, \mathbb{Z})$ taking the orthant $C$ and the sail $V(C)$ to themselves and establishing the isomorphism.

Unfortunately, the proof of this theorem is not yet published.

The algebraic version of the generalized Lagrange theorem was obtained by G. Lachaud. The formulation of his theorem requires special notation and definitions, so we just refer the reader to the article [22, since we will not use it further. For more information on generalizations of the Lagrange theorem for the ordinary continued fractions to the multidimensional case see the papers [19, 38], 22. and [24].

By a fundamental domain of a sail we mean the union of some faces that contains exactly one face from each equivalence class (with respect to the action of the group $\Xi(A))$.

0.2. Different algorithms for constructing the sails of multidimensional continued fractions. A multidimensional periodic algebraic continued fraction is a set of infinite polyhedral surfaces (i.e., sails) that contain an infinite number of faces. As we have already mentioned, the quotient of any sail under the Dirichlet group action is isomorphic to an $n$-dimensional torus. The algebraic periodicity of the polyhedron allows us to reconstruct the whole continued fraction knowing only the fundamental domain. Moreover, any fundamental domain contains only a finite number of faces of the whole algebraic periodic continued fraction. Hence we are faced with the problem of finding a good algorithm that enumerates all the faces for this domain.

There was no algorithm for constructing multidimensional continued fractions until T. Shintani's work 34] in 1976. Let $F$ be a totally real algebraic field of degree $n$. We take all different embeddings of $F$ into $\mathbb{R}$ and denote them by $\varphi_{i}$, 
$i=1, \ldots, n$ (there are exactly $n$ different embeddings, since $F$ is totally real). Consider the following embedding of $F$ into $\mathbb{R}^{n}$. For an arbitrary element $x$ of $F$ we suppose

$$
x \rightarrow\left(\varphi_{1}(x), \varphi_{2}(x), \ldots, \varphi_{n}(x)\right) .
$$

T. Shintani considered the action of the group of all totally positive elements for the ring of integers of $F$ (by componentwise multiplication by totally positive integers $\left.x_{+}\right)$on $\mathbb{R}_{+}^{n}$ for the described embedding of $F$. He proved that the fundamental domain for this action is the union of a finite number of simplicial cones of special type. (Note that if we take some other order for the embeddings $\varphi_{i^{\prime}}$, then the fundamental domains will be integer-linear equivalent to the fundamental domains for the embeddings considered above.) The statement of T. Shintani and its proof is actually the basis for the construction of one-dimensional continued fractions. Following T. Shintani's work, E. Thomas and A. T. Vasques obtained several fundamental domains for the two-dimensional case in [37. Finally, R. Okazaki in his article [29] presented a method that permits one to construct fundamental domains for fields of arbitrary degree. E. Korkina in [18], 20], 21] and G. Lachaud in 22, 23] produced an infinite number of fundamental domains for periodic algebraic two-dimensional continued fractions. The method used for constructing fundamental domains of multidimensional continued fractions in these papers was inductive. The method produces the fundamental domain face by face, verifying that each new face does not lie in the same orbit with some face constructed before. Applying this method, one can find the fundamental domain in finitely many steps.

Later on J. O. Moussafir developed an essentially different approach in 28. It works for an arbitrary (not necessarily periodic) continued fraction and computes any bounded part of an infinite polyhedron. The approach is based on deduction. One produces a conjecture on the face structure for a big part of the continued fraction, then it remains to prove that any conjectured face is indeed a face of the part. This method can be also applied to the case of periodic continued fractions.

In the present paper we describe a new advanced deductive construction adapted especially to fundamental domains of periodic continued fractions. The construction involves a method for conjecturing the structure of the fundamental domain and an algorithm testing whether the conjectured domain is indeed fundamental. The main advantage of our algorithm is the following: the number of "false" vertices of our approximation is much smaller than the number of "false" vertices of the approximation in the method of J. O. Moussafir (so that the computational time is considerably reduced).

Note that this algorithm substantially uses the periodicity of the continued fraction and hence it is impossible to apply it to non-periodic continued fractions.

We prove the following statement for the two-dimensional case.

Suppose we have a conjecture on the structure of the fundamental domain for some sail of a two-dimensional periodic continued fraction. Let this domain contain $N$ faces of all dimensions. The test of the conjecture (our algorithm) requires at most $C N^{4}$ additions, multiplications, and comparisons of two integers, where $C$ is a universal constant that does not depend on $N$.

All previous verification algorithms take exponential time with respect to $N$.

Remark 0.5. Here we do not take into account that the integers can be quite large. To calculate upper bounds for the running time of the algorithms we need 
to multiply upper bounds for the number of operations by some polynomial of the coefficients of the matrix defining the continued fraction.

Using the present algorithm, the author both generalized almost all known simple examples and series of examples of fundamental domains constructed before, and found many new examples and series (see [11] and [12]). Using these examples, the author found the complete list of all two-dimensional periodic continued fractions constructed by matrices of small norm $(|*| \leq 6)$ up to the integer-linear equivalence relation; see [13. By the norm of a matrix, here we mean the sum of the absolute values of all its coefficients.

0.3. Description of the paper. This work is organized as follows. The new method of sail construction consists of six steps. We discuss its plan in Section 1. In Section 2 we describe the two common steps for both inductive and deductive methods. In this section we show how to find the generators for the group of $S L(n, \mathbb{Z})$-matrices commuting with the given one. All results of Section 2 are well known and are given for completeness of exposition (see also Cohen [6] and G. Lachaud [23]). In Sections 3 and 4, we discuss the essential new part of the method. We show how to produce conjectures on fundamental domains in Section 3. In Section 4 we describe the algorithm for conjecture tests in the case of twodimensional continued fractions. In that section, we also say a few words about the higher dimensional case. We conclude in Section 5 with the detailed study of one example of the method's application (see also [11]).

\section{Description of the NeW COnStRuction}

1.1. Outline of the new construction. Now we briefly outline the main idea of the new construction of one of the fundamental domains of the multidimensional continued fraction corresponding to the given integer irreducible hyperbolic operator. Suppose that we are given the integer irreducible hyperbolic operator $A \in S L(n+1, \mathbb{Z})$. To compute some fundamental domain of a sail of the continued fraction associated to $A$ it is sufficient to do the following:

1. Compute a convex hull approximation of the sail. Namely take a large enough convenient set of integer points and find its convex hull.

2. Make a conjecture on some fundamental domain. Here we need to guess at a set of faces that might form a fundamental domain. We do this by finding a repeatable pattern in faces geometry.

3. Prove the conjecture if possible.

4. If you cannot prove the conjecture, start with 1 , but with a larger convenient set of points.

In this situation the following two questions are relevant:

How do we find a convenient set of integer points for the approximation of the sail?

How do we test whether the conjecture of a fundamental domain of the sail is true or not?

We give the answers to these questions in the present paper.

1.2. Steps of the construction. Let us briefly itemize the main steps of the method. 
The deductive algorithm for constructing one of the fundamental domains for the sail of the given operator $A$ in the given orthant.

Step 1. Calculate the basis of the additive group of the ring $\Xi(A)$.

Step 2. Calculate the basis of the group $\bar{\Xi}(A)$ (using the result of Step 1).

Step 3. Find some vertex of the sail.

Step 4. Make a conjecture on a fundamental domain of the sail (using the results of Step 2 and Step 3).

Step 5. Test the resulting (in Step 4) conjecture.

Remark 1.1. It is assumed that the fundamental domain conjectured in Step 5 and the basis $A_{1}, \ldots, A_{n}$ of the group $\bar{\Xi}(A)$ satisfy the following conditions:

i) the closure of the fundamental domain is homeomorphic to the disk;

ii) the operators $A_{1}, \ldots, A_{n}$ define the gluing of this disk to the $n$-dimensional torus.

Both inductive and deductive algorithms require the first and the second steps. We describe these two steps in the next section. All other steps are essential for our deductive algorithm. In 28 J.-O. Moussafir uses the approximation of the orthant by some rational orthant to produce a conjecture on a fundamental domain. In the present paper we propose to produce conjectures for some set of periods; see the description of Steps 3 and 4. We show how to test conjectures in the case of two-dimensional continued fractions in the description of Step 5. The result is partially based on the theorem on integer-affine classification of two-dimensional faces at the integer distances to the origin greater than one from [14]. (For the case of $n$-dimensional continued fractions for $n \geq 3$, the last step is quite complicated, since the classification of three-dimensional faces at integer distances to the origin greater than one is unknown.) In the last step we also investigate an important particular case that seems to be quite common for periodic $n$-dimensional continued fractions as well.

Remark 1.2. Note that all deductive algorithms are not algorithms in the strict sense. One should choose some basis of $\Xi(A)$ in the right way, produce a good conjecture, and then test it. Even the algorithmic recognition of the period for the given picture of the boundary of the sail approximation is thought to be a hard problem. That is the reason why this "algorithm" cannot be completed with certainty by some computer program. But on the other hand, the deductive algorithm is effective in practice. All of the examples listed in the article [11 were produced using this algorithm. The examples of this paper generalize and expand almost all known periods of the sails calculated before.

Remark 1.3. The present method can be naturally generalized to the case of the Minkowski-Voronoi model of multidimensional continued fractions. (For the definitions of the Minkowski-Voronoi model see [26] and 39].)

\section{General questions concerning the lattice bases}

In this section we briefly discuss the questions which are necessary for both inductive and deductive methods (Steps 1 and 2 of Section 1). The answers to these questions were known before (see also [23] and [6]). 
2.1. Step 1. Calculation of a basis of the additive group of the ring $\Xi(A)$. Let $V_{0} V_{1} \ldots V_{m}$ be some tetrahedron with vertices $V_{0}, V_{1}, \ldots, V_{m-1}$, and $V_{m}$. Denote by $P\left(V_{0}, V_{1}, \ldots, V_{m}\right)$ the following parallelepiped:

$$
\left\{V_{0}+\sum_{k=1}^{m} \alpha_{k} \overline{V_{0} V_{k}} \mid 0 \leq \alpha_{k} \leq 1, k=1, \ldots, m\right\} .
$$

In this section we consider $\Xi(A)$ as an additive group. We start the algorithm with the calculation of a basis for the group $\Xi(A)$. Let us identify the space $\operatorname{Mat}((n+1) \times(n+1), \mathbb{R})$ with the space $\mathbb{R}^{(n+1)^{2}}$ and consider the standard metrics for this space. So any integer operator corresponds to some integer point, and the distance between two operators is the Euclidean distance between the corresponding points in $\mathbb{R}^{(n+1)^{2}}$. We consider a sum of absolute values of all coefficients for some operator $A$ as a norm of the operator $A$ and denote it by $\|A\|$.

In Proposition 2.2 below we show that the set $\Xi(A)$ is an additive group isomorphic to $\mathbb{Z}^{n+1}$. Then by Corollary 2.3 below it follows that there exists a basis of the group $\Xi(A)$ contained in the parallelepiped $P\left(0, E, A, A^{2}, \ldots, A^{n}\right)$. Thus by Proposition 2.4 below all norms of elements of the basis are bounded above by

$$
N^{\prime}=\sum_{i=0}^{n}\left\|A^{i}\right\|
$$

Remark 2.1. Applying the LLL-algorithm described in 25 by A. K. Lenstra, H. W. Lenstra, Jr., and L. Lovász to the lattice generated by $0, E, A, A^{2}, \ldots, A^{n}$ one constructs some reduced basis. This will decrease the number $N^{\prime}$ to some number $N$. (It is not necessary to use LLL-algorithm here, just put $N=N^{\prime}$.)

The set of integer operators contained in the parallelepiped is also a subset of the following set:

$$
\operatorname{Mat}((n+1) \times(n+1), \mathbb{Z}) \cap B_{N}(O),
$$

where $B_{N}(O)$ is an $N$-neighborhood of the origin (i.e. the ball of radius $N$ centered at the origin). The last set contains at most $(2 N+1)^{n+1}$ elements. This set contains all integer operators of the parallelepiped $P\left(0, E, A, A^{2}, \ldots, A^{n}\right)$. Further, we find the set of integer points of the parallelepiped, this requires a polynomial in $N$ quantity of operations (we need to choose a subset of all points of the parallelepiped in the set considered above). Finally, we choose some basis of the group $\Xi(A)$ using the algorithm described in Proposition 2.5.

Now we formulate the statements mentioned above.

Proposition 2.2. For any integer irreducible hyperbolic operator $A$ the set $\Xi(A)$ forms an additive group isomorphic to $\mathbb{Z}^{n+1}$.

The detailed proof of Proposition 2.2 can be found, for instance, in the book 23. by G. Lachaud.

Corollary 2.3. There exists a basis of the group $\Xi(A)$, such that all its elements are contained in the parallelepiped $P\left(0, E, A, A^{2}, \ldots, A^{n}\right)$.

Proposition 2.4. Consider the parallelepiped $P\left(0, E, A, A^{2}, \ldots, A^{n}\right)$. The norms of all operators contained in this parallelepiped are bounded above by

$$
\sum_{i=0}^{n}\left\|A^{i}\right\|
$$

The proof is straightforward. 
Proposition 2.5. Suppose we are given a maximal rank sublattice of the integer lattice in the integer plane. Let $O ; A_{1}, \ldots, A_{n}$ generate this sublattice (here $O$ is the origin of the lattice). Then there exists a basis $O ; B_{1}, \ldots, B_{n}$ of the integer lattice in the parallelepiped $P\left(O, A_{1}, \ldots, A_{n}\right)$ such that for any natural $i \leq n$ the vertex $B_{i}$ belongs to the parallelepiped $P\left(O, A_{1}, \ldots, A_{i}\right)$.

Proof. We will inductively construct the basis $O ; B_{1}, \ldots, B_{n}$. On the $i$-th step we will construct the basis $O, B_{1}, \ldots, B_{i}$ inside the parallelepiped $P\left(O, A_{1}, \ldots, A_{i}\right)$ that satisfies all the conditions listed above for the lattice in the plane spanning the points $O, A_{1}, \ldots, A_{i}$.

Base of induction. For $i=1$ we choose the closest to the point $O$ integer point as $B_{1}$.

Step of induction. Suppose we have constructed vertices $O ; B_{1}, \ldots, B_{i-1}(i \leq n)$ satisfying the induction conditions. Now we construct an integer point $B_{i}$. Let the integer distance between the point $A_{i}$ and the plane spanned by the points $O, A_{1}, \ldots, A_{i-1}$ be equal to $d_{i-1}$. Consider the plane $\pi_{i}$ in the span of $O, A_{1}, \ldots, A_{i}$ that is parallel to the plane spanned by $O, A_{1}, \ldots, A_{i-1}$ and at unit integer distance to that plane. The plane $\pi_{i}$ is an integer plane. Therefore, the intersection of $\pi_{i}$ with the parallelepiped $P\left(O, A_{1}, \ldots, A_{i}\right)$ contains at least one integer point. We choose one of these points and denote it by $B_{i}$.

As far as the spans of $O, A_{1}, \ldots, A_{i-1}$ and $O, B_{1}, \ldots, B_{i-1}$ coincide, all integer points of the parallelepiped $P\left(O, B_{1}, \ldots, B_{i}\right)$ are at the integer distance 0 or 1 to the plane spanning $O, B_{1}, \ldots, B_{i-1}$. By the induction assumption the points $O ; B_{1}, \ldots, B_{i-1}$ generate the integer sublattice of the corresponding plane. Therefore, the parallelepiped $P\left(O, B_{1}, \ldots, B_{i-1}\right)$ is empty (i.e. does not contain integer points different from vertices of the parallelepiped). From the last two facts it follows that the parallelepiped $P\left(O, B_{1}, \ldots, B_{i}\right)$ is empty. Hence the points $O ; B_{1}, \ldots, B_{i}$ also generate the integer sublattice of the corresponding plane.

So we have constructed by induction a basis satisfying all the conditions of the proposition.

The algorithm of this step does not seem to be the optimal one. So the following question is natural here:

Problem 1. Find some effective algorithm for calculating an integer sublattice for the $k$-dimensional plane of the space $\mathbb{R}^{m}$ if some basis $A_{1}, \ldots, A_{k}$ for some sublattice of this plane is known (note that we know nothing about the corresponding quotient group).

2.2. Step 2. Calculation of a basis of $\bar{\Xi}(A)$. From the algorithmic point of view this step is the most complicated. We describe only the idea for one of the simplest algorithms here and give the corresponding references.

Let $\chi(x)$ be the characteristic polynomial of the operator $A$ and let $\xi$ be one of the roots of $\chi(x)$. Consider the following map

$$
h: \Xi(A) \rightarrow \mathbb{Q}[\xi] .
$$

For any element $B \in \Xi(A)$ there exists a unique representation $B=p_{B}(A)$, where the degree of the polynomial $p_{B}$ is less than $n+1$ (since the operators $E, A, \ldots A^{n}$ are linearly independent). We put

$$
h(B)=p_{B}(\xi) .
$$


Note that this map is an isomorphism between the ring $\Xi(A)$ and its image $h(\Xi(A))$. The addition and multiplication operations in the image are induced by the addition and multiplication operators of the field $\mathbb{Q}[\xi]$ (see $[23]$.). Moreover, the set $h(\Xi(A)$ ) forms an order in the field $\mathbb{Q}[\xi]$. By the Dirichlet unit theorem it follows that there exist a basis for the units of this order and a number $\rho$, such that the norms of all its elements are bounded above by $\rho$. Since the method of constructing the constant $\rho$ is standard, we omit it. (For the construction of $\rho$ and proofs we refer to 3 .) Note that the integer $(n+1)$-dimensional volume attains the minimal possible value at a simplex spanned by a basis operator. Now according to the book 3 we construct a basis by enumeration of all vectors of the set $h(\Xi(A))$ inside the ball $B_{\rho}(O)$, where $B_{\rho}(O)$ is a $\rho$-neighborhood of the origin. The preimage (i.e. $h^{-1}$ ) of this basis gives us a basis of the group of invertible elements in the ring $\Xi(A)$, and hence it gives a basis of the subgroup $\bar{\Xi}(A)$.

Remark 2.6. The constant $\rho$ is extremely large (it equals the exponent of some polynomial of the coefficients of the matrix $A$ ). An effective algorithm for this step can be found in the book [6] written by $\mathrm{H}$. Cohen. Using this algorithm one finds the basis of units in polynomial time (with respect to the coefficients of the matrix A).

Remark 2.7. Note that it is not necessary to find generators of $\bar{\Xi}(A)$. The algorithm works for arbitrary $n+1$ linearly independent operators of $\bar{\Xi}(A)$ (for more details see Remark 3.5 below).

\section{ON FUNDAMENTAL DOMAINS AND SAIL APPROXIMATIONS}

A basis of the group $\bar{\Xi}(A)$ was calculated in the previous section. Now we are coming to the main steps of the algorithm. In this section we calculate one of the sail vertices and show how to produce the conjectures.

3.1. Step 3. How to calculate one of the sail vertices. First let us find some integer point of the orthant containing the sail. Consider an arbitrary orthant. Shift the standard unit parallelepiped inside this orthant. Some integer point lies inside the shifted parallelepiped. The coordinates of this point coincide with integer parts of coordinates for one of $2^{n}$ vertices of this parallelepiped. Using this fact one can easily find such a point.

Having found some integer point $P$ of the orthant, let us find some vertex of the sail corresponding to this orthant. Consider some integer plane $\pi$ passing through the origin such that the intersection of $\pi$ with the orthant is at a unique point (at the origin). Suppose that the integer distance from the point $P$ to this plane is equal to $d$. Now we look through all the simplices obtained as intersections of our orthant with planes parallel to $\pi$ at integer distances to the origin equal to $1, \ldots, d$. Suppose that the first simplex containing integer points lies in the plane at integer distance $d^{\prime} \leq d$. The convex hull of all points of this simplex coincides with some faces of the sail. All vertices of this face are vertices of the sail. Choose an arbitrary one of them.

3.2. Step 4. How to produce a conjecture on the fundamental domain of a sail. Suppose that we know some point $V$ of the sail in the orthant, and a basis $A_{1}, \ldots, A_{n}$ for the group $\bar{\Xi}(A)$. Now we must produce a conjecture on a fundamental domain of the sail. Let us briefly describe how to do this. First, we compute 
the set of integer points that contains all vertices of some fundamental domain of the sail. Second, we show how to choose the infinite sequence of special polyhedron approximations for the sail. Finally, using a picture of these approximations, we formulate a conjecture on a fundamental domain of the sail.

Proposition 3.1. Let $V$ be a vertex of the sail of the $n$-dimensional continued fraction of an $(n+1)$-algebraic irrationality. Then there exists a fundamental domain of the sail such that all vertices of this domain are contained in the convex hull $H$ of the origin and of the $2^{n}$ distinct points of the form

$$
V_{\varepsilon_{1}, \ldots, \varepsilon_{n}}=\left(\prod_{i=1}^{n} A_{i}^{\varepsilon_{i}}\right)(V)
$$

where $\varepsilon_{i} \in\{0,1\}$ for $1 \leq i \leq n$.

Proof. Consider the polyhedral cone $C$ with vertex at the origin and base at the convex polyhedron with vertices $V_{\varepsilon_{1}, \ldots, \varepsilon_{n}}$. We take the union of all images of this polyhedral cone under the actions of the operators

$$
A_{m_{1}, \ldots, m_{n}}=\prod_{i=1}^{n} A_{i}^{m_{i}},
$$

for $1 \leq i \leq n$, where $m_{i} \in \mathbb{Z}$. Obviously, this union is equivalent to the union of the whole open orthant and the origin. Therefore, any vertex of the sail is obtained from a vertex contained in the cone $C$ by applying an operator $A_{m_{1}, \ldots, m_{n}}$ for some integers $m_{i}$. Moreover, the convex hull of all integer points of the given orthant contains the convex hull of the vertices of the form $A_{m_{1}, \ldots, m_{n}}(V)$. Hence the sail (i.e. the boundary of the convex hull of integer points) is contained in the closure of the complement in the orthant to the convex hull of all integer points of the form $A_{m_{1}, \ldots, m_{n}}(V)$. This complement is a subset of the union of polyhedra obtained from $H$ by an action of some operator $A_{m_{1}, \ldots, m_{n}}$ (for some integers $m_{i}, 1 \leq i \leq n$ ).

We skip the classical description of the computation of the convex hull for the integer points contained in the polyhedron $H$. Denote the vertices of this convex hull by $V_{r}$ for $0<r \leq N$. Here $N$ is the total number of such points.

Definition 3.2. The convex hull of the finite set of points

$$
\left\{A_{m_{1}, \ldots, m_{n}}\left(V_{r}\right) \mid 1 \leq m_{i} \leq m, \forall i: 0 \leq i \leq n\right\}
$$

is called the $n$-th special polyhedron approximation for the sail.

The defined set contains approximately, but less than, $m^{n} N$ points. (Since we calculated some image points for the boundary of $H$ several times, we do not know the exact number of points.) The number $N$ is fixed for the given generators $A_{1}, \ldots, A_{n}$ and $m$ varies. We should try to make a good conjecture with the least possible $m$.

Remark 3.3. For all the examples listed in the paper 11 (and for the example of the last section) it was sufficient to take $m=2$ to produce the corresponding conjectures.

Remark 3.4. Note that $N$ is a function defined on the set of all generators of the group $\bar{\Xi}(A)$ and it does not depend on $m$. Therefore, the "quality" of the approximation also depends on the choice of the basis. 
Remark 3.5. Now we briefly discuss the case of Remark 2.7. Suppose we know operators $A_{1}, \ldots, A_{n}$ that generate only some full rank subgroup of the group $\bar{\Xi}(A)$. Let the index of this subgroup be equal to $k$. Then we are faced with the following two problems. First, the number $N$ will be approximately $k$ times greater than in the previous case. Second, one should also find a conjecture on generators of the group $\bar{\Xi}(A)$.

\section{Test of the ConjeCtures Produced in the two-Dimensional CASE}

Now it remains to test the produced conjectures of Step 4. In this section we explain how to test conjectures for the case of two-dimensional periodic continued fractions. The test consists of seven stages. It uses the classification theorem from [14. We prove here that these seven stages are sufficient for the verification of whether the conjecture produced is true or not. The complexity of these stages is polynomial in the quantity of all faces.

4.1. Brief description of the test stages and formulation of the main results. Suppose we have a conjecture on some fundamental domain $D$ for some sail of a two-dimensional periodic continued fraction associated to some integer irreducible hyperbolic operator $A$. Also from Step 2 we know some basis $B_{1}, B_{2}$ of the group $\bar{\Xi}(A)$. Let $p_{k}$ (for $k=0,1,2$ ) be the number of $k$-dimensional faces of the fundamental domain $D$. Denote by $F_{i}\left(i=1, \ldots, p_{2}\right)$ the two-dimensional faces, i.e. polygons. All vertices and edges adjacent to each face are known. It is also conjectured that the fundamental domain $D$ and the basis $B_{1}, B_{2}$ satisfy the following conditions:

i) the closure of the fundamental domain is homeomorphic to the two-dimensional disk;

ii) $B_{1}$ and $B_{2}$ define the gluing of this disk to the $n$-dimensional torus (the fundamental domain $D$ is in one-to-one correspondence with this torus).

Test of the conjecture. Our test consists of the following seven stages:

1. test of condition i);

2. test of condition ii);

3. calculation of all integer distances from the origin to the two-dimensional planes containing faces $F_{i}$ and verification of their positivity;

4. test the nonexistence of integer points inside the pyramids with vertices at the origin and bases at $F_{i}$ (here the integer points of faces $F_{i}$ are permitted);

5 . test the convexity of dihedral angles (for all edges of the fundamental domain);

6. verification that all stars of the vertices are regular;

7. test whether all vertices of $D$ are in the same orthant.

Theorem 4.1. The described conjecture test for the fundamental domain $D$ requires less than

$$
C\left(p_{0}+p_{1}+p_{2}\right)^{4}
$$

additions, multiplications, and comparisons of two integers, where $C$ is a universal constant that does not depend on the $p_{i}$.

Remark 4.2. Note that here we do not take into account the complexity of additions, multiplications, and comparison of two large integers. We think of any such operation as a single operation (as a unit of time). There are some known bounds 
for the number of digits of the integers that are linear with respect to the coefficients of the matrix $A$. So the complexity should be multiplied by some polynomial in the coefficients of $A$.

Theorem 4.3. Let the set of faces D satisfy the following conditions:

1) condition i);

2) condition ii);

3) positivity of all integer distances from the origin to the two-dimensional planes containing faces $F_{i}$;

4) there are no integer points inside the pyramids with vertices at the origin and bases at $F_{i}$ (here integer points on the faces $F_{i}$ are permitted);

5) all dihedral angles are convex;

6) all stars of the vertices are regular;

7) all vertices of $D$ are contained in the same orthant.

Then $D$ is a fundamental domain of some sail of the continued fraction associated to the operator $A$.

We start with the proof of Theorem 4.1. Let us show that all the stages listed above can be executed in polynomial time.

4.2. Test of condition i). First, we need to test that the closures of any two two-dimensional faces either do not intersect or intersect at a vertex, or at an edge (and hence the closures of any two one-dimensional faces either do not intersect or intersect at a vertex). For this test we need to solve linear systems of two equations and $2 p_{1}$ inequalities that define faces $F_{i}$ (in three variables). The number of such systems equals the number of couples of faces, i.e., equals $\frac{p_{2}\left(p_{2}-1\right)}{2}$. Since there are only three variables and two linear equalities, any system can be reduced to a system of inequalities in one variable in time linear in $p_{1}$. This implies that we need at most $C_{1,1} p_{1} p_{2}^{2}$ operations to solve all systems, where $C_{1,1}$ is some constant that does not depend on $p_{i}$.

Second, we have to test that the edges are adjacent either to one or to two (two-dimensional) faces. This can be done in less than $p_{1} p_{2}$ single adjacency tests. Any adjacent test can be done in time linear in $p_{1}$. This yields that we need at most $C_{1,2} p_{1}^{2} p_{2}$ operations for these tests, where $C_{1,2}$ is some constant that does not depend on $p_{i}$.

Consider all (one-dimensional) edges of the closure of $D$ that are adjacent to exactly one face of $D$. Third, we test that the union of such edges is homeomorphic to the circle, i.e., is piecewise connected and does not have self-intersections. We will check that this set is piecewise connected, and that for any vertex of the closure of $D$ either exactly two of the described edges are adjacent to the vertex or none of the described edges are adjacent to it. The test contains at most $p_{1}^{2}$ simple adjacency tests. Any simple adjacency test requires a finite number of operations that does not depend on $p_{i}$ for $i=0,1,2$. So, we need at most $C_{1,3} p_{1}^{2}$ operations for these tests, where $C_{1,3}$ is some constant that does not depend on the $p_{i}$.

Further, we will check that the union of the closures of all faces of $D$ is piecewise connected. Let us find explicitly one of the connected components. Consider an arbitrary face and all its edges and vertices. Take all faces whose closures contain these edges and vertices (except the first face) and consider all new edges and vertices at their boundaries. We continue our construction inductively. Suppose we have performed $l$ steps and constructed some part of the connected component. 
Consider again all new (appearing at the $i$-th step) edges and vertices on its boundary. Take all faces whose closures contain these edges and vertices (except the old faces). Since any edge of $D$ considered at most once, this algorithm requires at most $C_{1,4}^{\prime} p_{1}$ simple adjacency tests. So we know one of the connected components. Now it remains to check that all faces are in this component and all edges and vertices are adjacent to these faces. In total we need at most $C_{1,4}\left(p_{0}+p_{1}+p_{2}\right)$ operations.

Suppose all tests of this subsection are positive. So the closure of $D$ is piecewise connected, and its boundary is homeomorphic to the circle. The closure of $D$ is homeomorphic to the two-dimensional disk iff its Euler characteristic equals one. We need less than $4 p_{0}+2 p_{1}+p_{2}$ additions for the calculation of Euler characteristics for the closure of $D$.

We have proved the following lemma.

Lemma 4.4. The first stage of the test requires at most

$$
C_{1}\left(p_{1}^{2} p_{2}+p_{1} p_{2}^{2}+p_{0}\right)
$$

additions, multiplications, and comparisons of two integers, where $C_{1}$ is a universal constant that does not depend on $p_{i}$.

4.3. Test of condition ii). First we check that after the gluing of the disk $D$ we obtain a nonsingular triangulated surface.

Let us check that there is no singular points inside the disk, i.e., open faces of the interior of the disk and their images after gluing do not intersect. This requires $2\left(p_{0}+p_{1}+p_{2}\right)^{2}$ systems of linear equations and inequalities. Any such system can be solved in a linear time with respect to $p_{0}, p_{1}, p_{2}$. Hence we need at most $C_{2,1}\left(p_{0}+p_{1}+p_{2}\right)^{3}$ operations, where $C_{2,1}$ is a universal constant that does not depend on $p_{i}$.

Now we check that there are no singularities on edges on the boundary of the disk. Here we need to check that for any edge of the boundary there exists a unique edge that is glued to the first edge. This requires at most $p_{1}^{2}$ adjacency tests in $C_{2,2}$ operations each. Hence we need at most $C_{2,2} p_{1}^{2}$ operations $\left(C_{2,1}\right.$ does not depend on $p_{i}$ ).

Further, we check that there are no singularities at the vertices (after the gluing). For any vertex we need to check that the union of all faces and edges containing the vertex in their closure is homeomorphic to the disk. Now we know that faces of the fundamental domain do not intersect. It remains only to check that any edge is adjacent to exactly two faces (after gluing), and that the union of all faces and edges containing the vertex in their closure is piecewise connected and orientable. The test of all these conditions requires at most $h p_{1} p_{2}$ adjacency tests for some constant $h$. Thus to test a vertex we need at most $C_{2,3} p_{1} p_{2}^{2}$ operations $\left(C_{2,3}\right.$ does not depend on $p_{i}$ ). For all vertices of the boundary we need at most $C_{2,3} p_{0} p_{1} p_{2}^{2}$ operations.

Finally, let us check that this surface is homeomorphic to the torus. First the Euler characteristic of the surface should be zero (to verify this we need a time linear in $p_{i}$ ). Second we show the orientability of the surface. We orient the boundary circle and check that any two boundary edges that we glue together are glued with the opposite orientations. If for one of the pairs of such edges the orientation coincides, we have a Klein bottle. Otherwise, we get a torus. This requires a number of operations linear in $p_{1}$. Therefore, for this test we need at most $C_{2,4}\left(p_{0}+p_{1}+p_{2}\right)$ operations $\left(C_{2,4}\right.$ does not depend on $\left.p_{i}\right)$. 
It remains to show that the fundamental domain $D$ bijectively maps to the torus we obtained. For all faces this holds automatically. The corresponding test for edges and vertices requires $C_{2,5}\left(p_{0}+p_{1}\right)$ operations $\left(C_{2,5}\right.$ does not depend on $\left.p_{i}\right)$.

We have proven the following lemma.

Lemma 4.5. The second stage of the test requires at most

$$
C_{2}\left(p_{0} p_{1}^{2} p_{2}+\left(p_{0}+p_{1}+p_{2}\right)^{3}\right)
$$

additions, multiplications, and comparisons of two integers, where $C_{2}$ is a universal constant that does not depend on $p_{i}$.

4.4. Calculation of all integer distances from the origin to the two-dimensional planes containing faces $F_{i}$ and their positivity. Let $V_{1}\left(x_{1}, y_{1}, z_{1}\right)$, $V_{2}\left(x_{2}, y_{2}, z_{2}\right)$ and $V_{3}\left(x_{3}, y_{3}, z_{3}\right)$ be some integer points that do not lie in a straight line. Then the following statement holds.

Proposition 4.6. The integer distance from the origin to the two-dimensional integer plane that spans the points $V_{1}, V_{2}$, and $V_{3}$ is equal to

$$
\frac{\left|\left(\begin{array}{lll}
x_{1} & x_{2} & x_{3} \\
y_{1} & y_{2} & y_{3} \\
z_{1} & z_{2} & z_{3}
\end{array}\right)\right|}{\left|\left[\overline{V_{2}-V_{1}}, \overline{V_{3}-V_{1}}\right]\right|_{\mathbb{Z}}},
$$

where by $\left[\overline{V_{2}-V_{1}}, \overline{V_{3}-V_{1}}\right]$ we denote the cross product of the vectors $\overline{V_{2}-V_{1}}$ and $\overline{V_{3}-V_{1}}$ in $\mathbb{R}^{3}$, and by $|V|_{\mathbb{Z}}$ we denote the integer length of the vector $V$ (i.e. the greater common divisor of the coordinates of this vector).

The proof is straightforward and is omitted.

Remark 4.7. This proposition can be generalized to the higher dimensional case.

Lemma 4.8. The third stage of the test requires at most $C_{3} p_{2}$ additions, multiplications, and comparisons of two integers, where $C_{3}$ is a universal constant that does not depend on $p_{i}$.

Proof. Any integer distance to the origin can be found by the formula of Proposition 4.6 (this requires some finite number of steps that does not depend on $p_{i}$ ).

4.5. Test of the nonexistence of integer points inside the pyramids with vertices at the origin and bases at $F_{i}$. First we formulate the following integerlinear classification theorem.

Theorem 4.9 (see [14]). Any compact two-dimensional face of a sail of a twodimensional continued fraction contained in a plane at integer distance to the origin greater than one is integer-linear equivalent exactly to one of the faces with vertices of the following list:

$-(\xi, r-1,-r),(a+\xi, r-1,-r),(\xi, r,-r)$, where $a \geq 1$, and $\xi$ and $r$ are relatively prime, and $r \geq 2$ and $0<\xi \leq r / 2$;

- $(2,1, b-1),(2,2,-1),(2,0,-1)$, where $b \geq 2$;

- $(2,-2,1),(2,-1,-1),(2,1,2)$ and $(3,0,2),(3,1,1),(3,2,3)$.

For this stage we will also need the following proposition. 
Proposition 4.10. Consider two integer triangles in $\mathbb{R}^{3}$ with vertices $A_{1}\left(x_{a_{1}}\right.$, $\left.y_{a_{1}}, z_{a_{1}}\right), A_{2}\left(x_{a_{2}}, y_{a_{2}}, z_{a_{2}}\right), A_{3}\left(x_{a_{3}}, y_{a_{3}}, z_{a_{3}}\right)$ and with vertices $B_{1}\left(x_{b_{1}}, y_{b_{1}}, z_{b_{1}}\right)$, $B_{2}\left(x_{b_{2}}, y_{b_{2}}, z_{b_{2}}\right), B_{3}\left(x_{b_{3}}, y_{b_{3}}, z_{b_{3}}\right)$. Suppose that the plane of the first triangle does not contain the origin. Then the triangle $A_{1} A_{2} A_{3}$ is integer-linear equivalent to the triangle $B_{1} B_{2} B_{3}$ (in the corresponding order) iff the absolute value of the determinant of the matrix $B A^{-1}$ equals one, and all the coefficients of this matrix are integers, where

$$
A=\left(\begin{array}{ccc}
x_{a_{1}} & x_{a_{2}} & x_{a_{3}} \\
y_{a_{1}} & y_{a_{2}} & y_{a_{3}} \\
z_{a_{1}} & z_{a_{2}} & z_{a_{3}}
\end{array}\right), \quad B=\left(\begin{array}{lll}
x_{b_{1}} & x_{b_{2}} & x_{b_{3}} \\
y_{b_{1}} & y_{b_{2}} & y_{b_{3}} \\
z_{b_{1}} & z_{b_{2}} & z_{b_{3}}
\end{array}\right) .
$$

We leave the proof of this proposition as an easy exercise to the reader.

Lemma 4.11. The fourth stage of the test requires at most $C_{4} p_{2}$ additions, multiplications, and comparisons of two integers, where $C_{4}$ is a universal constant that does not depend on $p_{i}$.

Proof. Consider some face $F_{i}$. If the integer distance from the origin to the plane containing the face equals one, then all integer points of the pyramid except the vertex at the origin are contained in the base.

Suppose now that the integer distance from the origin to the plane containing the face equals $r_{i}>1$. By Theorem 4.9 it follows that this pyramid is triangular. First we calculate integer lengths of the edges and the integer area of $F_{i}$ (in a fixed number of operations). Further, by Proposition 4.10 the verification of an integerlinear type of the face is reduced to the solution of nine integer-linear equations on $\xi$ or to the verification that some nine rationals are integers.

Therefore, for any face we need some constant number of operations $C_{4}$ (where $C_{4}$ does not depend on $p_{i}$ ). Thus the complexity of the fourth stage of the test is at most $C_{4} p_{2}$.

4.6. Test of the convexity of dihedral angles. A dihedral angle is called wellplaced if the origin is contained in the corresponding opposite angle.

Note that the property of some angle being well-placed is an integer-linear invariant. Thus it is sufficient to check this property for all dihedral angles for edges of the closure of $D$. The test for each edge reduces to the solution of a system of ordinary inequalities (without variables).

Lemma 4.12. The fifth stage of the test requires at most $C_{5} p_{2}^{2}$ additions, multiplications, and comparisons of two integers, where $C_{5}$ is a universal constant that does not depend on $p_{i}$.

4.7. Verification that all 2-stars of the vertices are regular. Let $p: W \rightarrow T^{2}$ be the universal covering of the torus after gluing. We define the 2 -star at the vertex of the universal covering of the torus to be the union of all faces of the universal covering (of dimensions no more than 2) to which the given vertex is adjacent.

Let a vertex $v \in W$ map to $x \in \mathbb{R}^{3}$. The faces of the universal covering to which the given vertex is adjacent maps to faces with the same property. If $x \neq(a, 0,0)$ for some positive $a$, then by $\bar{v}_{n}$ we denote the vector $(1 / n, 0,0), n \in \mathbb{N}$. (If $x=(a, 0,0)$, then by $\bar{v}_{n}$ we denote $(0,1 / n, 0)$.) A 2 -star at $v$ is called regular if for the sequence of rays $l_{n}$ passing through the point $x+\bar{v}_{n}$ and having vertexes at the origin there exists a positive $k$ such that for any $m \geq k$ the following holds: the preimage $\left(p^{-1}\right)$ 
of the intersection of the image of 2-star at $v$ and $l_{m}$ in the universal covering consists of exactly one point.

Lemma 4.13. The sixth stage of the test requires at most

$$
C_{6} p_{0} p_{1}\left(p_{0}+p_{2}\right)
$$

additions, multiplications and comparisons of two integers, where $C_{6}$ is a universal constant that does not depend on $p_{i}$.

Proof. For any face (edge) of the image of the 2 -star for any vertex we need to solve a system of at most $p_{1}$ ( $p_{0}$, respectively) linear inequalities of the variable $\varepsilon=1 / n$. Hence the complexity of the test is at most

$$
C_{6} p_{0}\left(p_{0} p_{1}+p_{1} p_{2}\right),
$$

where $C_{6}$ is a universal constant that does not depend on $p_{i}$.

\subsection{Test for all vertices of $D$ to be in the same orthant: Conclusion of the proof of Theorem 4.1 .}

Lemma 4.14. The seventh stage of the test requires at most $C_{7} p_{0}$ additions, multiplications and comparisons of two integers, where $C_{7}$ is a universal constant that does not depend on $p_{i}$.

Proof. We will use the following statement. Let $e$ be some nonzero vector. The volume of the parallelepiped generated by the vectors $e, A(e)$, and $A^{2}(e)$ equals zero iff the vectors $e, A(e)$, and $A^{2}(e)$ generate some eigensubspace of $A$ (of nonzero codimension).

Consider two vertices $x_{1}$ and $x_{2}$ of $D$. Let $x(t)=t x_{1}+(1-t) x_{2}$. The vertices $x_{1}$ and $x_{2}$ are contained in the same orthant iff the volume function $f(t)$ of the parallelepiped generated by the vertices $x(t), B_{1}(x(t))$, and $B_{1}^{2}(x(t))$ does not have zeros in the segment $[0,1]$. Note that $f(x)$ equals the determinant of the matrix generated by the vectors $x(t), B_{1}(x(t))$, and $B_{1}^{2}(x(t))$. Thus $f(x)$ is a polynomial of the third degree with integer coefficients. All solutions of $f(x)=0$ can be found explicitly. It remains to compare them with 0 and 1.

Let us fix $x_{1}$ and vary $x_{2}$ (in the set of all vertices of $D$ ). The corresponding test requires at most $C_{7} p_{0}$ operations $\left(C_{7}\right.$ does not depend on $\left.p_{i}\right)$.

Remark 4.15. As long as the integer operator $B_{1}$ is hyperbolic and irreducible and all the coefficients of $f(x)$ are integers, it follows that $f(x)$ has three distinct real roots. So the question of the existence of roots in $[0,1]$ can be reduced to calculating critical points, comparing them with 0 and 1 , and comparing the critical values with $f(0)$ and $f(1)$. Here we solve only one quadratic equation (instead of the cubic one $f(x)=0)$.

Conclusion of the proof of Theorem 4.1. From Lemmas 4.4 4.14 it follows that all seven stages of the test require at most $C\left(p_{0}+p_{1}+p_{2}\right)^{4}$ additions, multiplications, and comparisons of two integers, where $C$ is a universal constant that does not depend on $p_{i}$.

Remark 4.16. Actually a stronger statement holds. The whole test requires no more than $\bar{C}\left(p_{0}+p_{1}+p_{2}\right)^{3}$ additions, multiplications and comparisons of two integers, and $\tilde{C}\left(p_{0}+p_{1}+p_{2}\right)^{4}$ logical operations, where $\bar{C}$ and $\tilde{C}$ are universal constants that do not depend on $p_{i}$.

It remains to prove that these seven stages are sufficient for the test. 
4.9. Lemma on the injectivity of the face projection. We prove Theorem 4.3 in four lemmas.

First let us give the necessary notation. Let the operators $B_{1}$ and $B_{2}$ generate $\Xi(A)$. For any integers $n, m$ we write $B_{n, m}$ for the operator $B_{1}^{n} B_{2}^{m}$. We suppose that our domain $D$ satisfies conditions $1-7$ of Theorem 4.3 , Let

$$
U=\bigcup_{n, m \in \mathbb{Z}} B_{n, m}(D) .
$$

Consider the two-dimensional unit sphere $S^{2}$ centered at the origin $O$. We denote by $\pi$ the following map:

$$
\pi: \mathbb{R}^{3} \backslash O \rightarrow S
$$

where any point $x \in \mathbb{R}^{3} \backslash O$ maps to the point at the intersection of $S^{2}$ and the ray with vertex at the origin and containing $x$.

Lemma 4.17. For any face of the polygonal surface $U$ the map $\pi$ is well defined and injective on it.

Proof. Consider any two-dimensional face $F$ of the surface $U$. By condition 3, the distance from the origin to the plane containing $F$ is greater than zero. Hence this plane does not contain the origin. Then $\pi$ is well defined and injective on $F$.

Now let $E$ be some edge of $U$. By conditions 1 and 2, this edge is adjacent to some two-dimensional face and therefore is contained in some plane that does not pass through the origin. Thus the line containing $E$ does not pass through the origin. Hence $\pi$ is well defined and injective on $E$.

The injectivity for the vertices is obvious.

4.10. Lemma on the finite covering of the fundamental domain. Let $x \in$ $\mathbb{R}^{3} \backslash O$. Denote by $N_{x}$ the tetrahedral angle with vertex at the origin and base with vertices $x, B_{1}(x), B_{1} B_{2}(x)$, and $B_{2}(x)$. Notice that

$$
\left(\bigcup_{n, m \in \mathbb{Z}} B_{n, m}\left(N_{x}\right)\right) \backslash O
$$

is one of eight orthants of the continued fraction associated to $A$ that contains $x$. Note that from conditions 1,2 , and 6 it follows that all points of $D$ are contained in one open orthant which we denote by $K$.

Lemma 4.18. Let $x$ be some point of the open orthant $K$. Then the union of all faces of $D$ is contained in a finite union of solid angles of the type $B_{n, m}\left(N_{x}\right)$.

Proof. By the Dirichlet unit theorem [3] it follows that for any interior point $a$ of the open orthant $K$ there exists an open neighborhood satisfying the following condition. The neighborhood can be covered by four solid angles of the type $B_{n, m}\left(N_{x}\right)$ when $a$ belongs to an edge of some $B_{k, l}\left(N_{x}\right)$; by two solid angles when $a$ belongs to the face of some $B_{k, l}\left(N_{x}\right)$; and by one solid angle in the remaining cases. In any case the neighborhood can be covered by some finite union of solid angles of the type $B_{n, m}\left(N_{x}\right)$.

Consider a covering of $D$ by such neighborhoods that correspond to each point of the closure of $D$. Since the closure of $D$ is closed and bounded in $\mathbb{R}^{3}$, it is compact. Hence this covering contains some finite subcovering. Therefore the union of all faces of $D$ is contained in the finite union of solid angles of type $B_{n, m}\left(N_{x}\right)$. 
Corollary 4.19. Let $x$ be contained in the open orthant $K$. Then the solid angle $N_{x}$ contains only points from a finite number of fundamental domains of the type $B_{n, m}(D)$.

Proof. From the last lemma it follows that $D$ is contained in the finite union $\bigcup_{k=1}^{l} B_{n_{k}, m_{k}}\left(N_{x}\right)$ (for some positive $l$ ). Then the solid angle $N_{x}$ can contain only points of the fundamental domains $B_{-n_{k},-m_{k}}(D)$ for $1 \leq k \leq l$.

\subsection{Lemma on the bijectivity of the projection.}

Lemma 4.20. The map $\pi$ bijectively takes the polygonal surface $U$ to the set $S^{2} \cap K$.

Proof. As was shown above, the surface $U$ is contained in $K$ and is taken to $S^{2} \cap K$ under the map $\pi$.

Let us introduce the following notation. By condition 2, the action of the operators $B_{1}$ and $B_{2}$ determine a gluing of the fundamental domain. After gluing we obtain a torus, denoted by $T^{2}$. Let $W$ be the universal covering of $T^{2}$. The face decomposition on $T^{2}$ lifts to a face decomposition on $W$. There is a natural two-parameter family (with two integer parameters) of projections $p_{n, m}: W \rightarrow U$ that maps faces to faces (since the group of shifts $B_{k, l}$ acts on $U$ ). Let us choose one of these projections and denote it by $p(p: W \rightarrow U)$.

Consider the map $\pi \circ p: W \rightarrow S^{2}$. This map does not have branch points at the images of open faces of $W$, since any face of $W$ bijectively maps to some face of $U$, and the corresponding face of $U$ injectively maps to $S^{2} \cap K$ by Lemma 4.17 .

Two faces with a common edge of the universal covering $W$ map to some two faces with a common edge of the surface $U$, such faces of $U$ generate a well-placed dihedral angle, and hence also injectively map to $S^{2} \cap K$. So the map $\pi \circ p$ does not have branch points at the images of open edges.

We now consider some vertex $v$ of $W$. The edges and faces of $W$ with common vertex $v$ by condition 6 form a regular 2-star. These edges also map to some edges of $U$ with common vertex. Thus there exists a sequence of points that tend to $\pi \circ p(v)$ (contained in $S^{2}$ ) such that the preimage of any point of the sequence has exactly one preimage in the 2-star of $v$. Hence $\pi \circ p$ does not have branch points in the sheet containing the star at $\pi \circ p(v)$. Therefore, $\pi \circ p$ does not have any branch points at the vertices. So the map $\pi \circ p: W \rightarrow S^{2} \cap K$ does not have branch points.

Consider an arbitrary point $x \in S^{2} \cap K$ and the solid angle $N_{x}$ corresponding to it. Let $x_{1}$ and $x_{2}$ be two points of $S^{2} \cap N_{x}$. We will now show that the preimages $(\pi \circ p)^{-1}\left(x_{1}\right)$ and $(\pi \circ p)^{-1}\left(x_{2}\right)$ contain the same number of points. Let us join the points $x_{1}$ and $x_{2}$ by some curve inside $S^{2} \cap N_{x}$. By Corollary 4.19, we know that the preimage of this curve is contained in a finite number of faces of $W$. Since there are no branch points in any face (and their number is finite) and there are no boundary faces of $W$, the number of preimages for $\pi \circ p$ is some (finite) discrete and continuous function on this curve. Therefore, the number of preimages for $\pi \circ p$ of any two points of $S^{2} \cap N_{x}$ is the same. Hence the number of preimages for $\pi \circ p$ of any two points of $S^{2} \cap K$ is the same.

From this we conclude that the projection $\pi \circ p$ of the universal covering $W$ (homeomorphic to an open disk) to $S^{2} \cap K$ (i.e. homeomorphic to an open disk) is a nonramified covering with finitely many sheets. Since the covering (of an open disk by an open disk) is piecewise connected, the number of sheets equals one. Since by definition $p: W \rightarrow U$ is surjective and by all of the above it is injective, the maps $p: W \rightarrow U$ and $\pi: U \rightarrow S^{2} \cap K$ are bijective. 
4.12. Lemma on convexity. Since any ball centered at the origin contains only a finite number of vertices of $U$ (and they do not form a sequence tending to the boundary of the orthant $K$ ), the polyhedral surface $U$ divides the space $\mathbb{R}^{3}$ into two connected components. Denote by $H$ the connected component of the complement to $U$ that does not contain the origin.

Lemma 4.21. The set $H$ is convex.

Proof. Suppose that some plane passing through the origin intersects the polygonal surface $U$ and does not contain any vertex of $U$. By Lemma 4.20 such a plane intersects $U$ at some piecewise-connected broken line with an infinite number of edges. The complement of the plane of this broken line consists of two connected components. By assumption all vertices of this broken line are contained in open edges of $U$. By condition 5 all dihedral angles of $U$ are well placed. Thus the angle at any vertex of intersection of $H$ with our plane is less than a straight angle. Hence by the previous lemma the intersection is convex.

Consider the set of all planes that pass through the origin, intersect $U$, and do not contain vertices of $U$. This set is dense in the set of all planes passing through the origin and intersecting $U$. Therefore, by continuity it follows that the intersection of $H$ with any plane passing through the origin (and intersecting $U$ ) is convex.

Now we prove that the set $H$ is convex. Let $x_{1}$ and $x_{2}$ be some points of $H$. Consider the plane that spans $x_{1}, x_{2}$ and the origin. This plane intersects $U$ since $x_{1}$ is in $H$ and the origin is not in $H$. By the above the intersection of $H$ with this plane is convex. Hence the segment with endpoints $x_{1}$ and $x_{2}$ is contained in $H$. Thus $H$ is convex (by the definition of convexity).

4.13. Conclusion of the proof of Theorem 4.3; The main part. So the constructed polygonal surface $U$ has the following properties:

— by Lemma $4.21 U$ bounds the convex set $H$;

- by construction all vertices of $U$ are integer points;

- by condition 4 the set $K \backslash H$ does not contain integer points.

Therefore, the polygonal surface $U$ is the boundary of the convex hull of all integer points inside $K$. Thus by definition $U$ is one of the sails of the continued fraction associated to the operator $A$.

Let us formulate one important conjecture here.

Conjecture 4.22. Conditions 1-6 imply condition $\%$.

4.14. On the verification of the conjecture for the multidimensional case. Here we briefly outline an idea of how to test the conjecture for fundamental domains of multidimensional continued fractions.

Conjecture for the multidimensional case. Suppose we have a conjecture on some fundamental domain $D$, and also some basis $B_{1}, \ldots, B_{n}$ of the group $\bar{\Xi}(A)$. Also, let the fundamental domain and the basis have the following properties:

i) the closure of the fundamental domain is homeomorphic to the disk;

ii) the operators $B_{1}, \ldots, B_{n}$ define the gluing of this disk to the $n$-dimensional torus.

How do we test the conjecture for fundamental domains of multidimensional continued fractions? The verification of conditions i) and ii) is straightforward and is omitted. If these conditions hold we check whether all the $n$-dimensional faces 
of the fundamental domain are faces of the sail. This can be done in the following way.

Suppose that the integer distances from the origin to the planes of faces $F_{i}$ are equal to $d_{i}(i=1, \ldots, p$, where $p$ is the number of all $n$-dimensional faces). Our conjecture is true if and only if for all $i=1, \ldots, p$ the following conditions hold:

a) For any integer $d<d_{i}$ consider the plane parallel to the face $F_{i}$ with integer distances to the origin equal to $d$. The intersection of our orthant with this plane does not contain any integer point.

b) For $d=d_{i}$ the convex hull of all integer points in the intersection coincides with face $F_{i}$.

The verification of conditions a) and b) is quite complicated from the algorithmical point of view.

We conclude this section with the important inverse question of constructing periodic continued fractions.

Question 3. (V. I. Arnold.) Does there exist an algorithm to decide whether a given type of fundamental domain is realizable by a periodic continued fraction?

The answer to this question is unknown even for the two-dimensional periodic continued fractions.

\section{An eXAmple of the CAlCUlation of a FUndamental Domain}

The example of two-dimensional sails in this section was announced in my article [11. For arbitrary integer numbers $m$ and $n$ we denote by $A_{m, n}$ the Sylvester operator

$$
\left(\begin{array}{ccc}
0 & 1 & 0 \\
0 & 0 & 1 \\
1 & -m & -n
\end{array}\right) .
$$

We construct fundamental domains for some particular two-dimensional subfamily of the two-dimensional continued fractions corresponding to Sylvester operators for the orthant that contains the point $(0,0,1)$.

Theorem 5.1. Let $m=b-a-1, n=(a+2)(b+1)$ (where $a, b \geq 0)$. Consider the sail of the operator $A_{m, n}$ containing the point $(0,0,1)$. Let $A=(1,0, a+2)$, $B=(0,0,1), C=(b-a-1,1,0)$, and $D=\left((b+1)^{2}, b+1,1\right)$. Then the following set of faces forms one of the fundamental domains:

1) the vertex $A$;

2) the edges $A B, A D$, and $B D$;

3) the triangular faces $A B D$ and $B D C$.

The closure of the fundamental domain is homeomorphic to the square shown on Figure 1 (for the case of an arbitrary $a$, and $b=6$ ).

Steps 1 and 2. We omit the first and the second steps (these steps are classical, see [6]) and here write down the result. The following two operators generate the group $\bar{\Xi}(A)$ :

$$
X_{a, b}=A_{m, n}^{-2}, \quad Y_{a, b}=A_{m, n}^{-1}\left(A_{m, n}^{-1}-(b+1) I\right),
$$

where $I$ is the identity element in the group $S L(3, \mathbb{Z})$.

Step 3. We prove that $(0,0,1)$ is a vertex of the sail. Consider the plane passing through $A, B$, and $D$ :

$$
(-1-a) x+(a b+a+b+1) y+z=1 .
$$




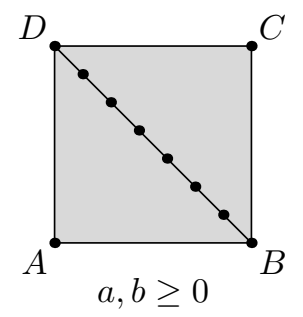

Figure 1. The closure of the fundamental domain of a sail of a fraction associated to the operator $A_{b-a-1,(a+2)(b+1)}$ (here $b=6$, and $a$ is arbitrary).

When the equations (in variables $x, y$, and $z$ )

$$
(-1-a) x+(a b+a+b+1) y+z=\alpha
$$

do not have any integer solution for $0<\alpha<1$, the integer distance from $A B D$ to the origin is equal to one. There are exactly three integer points $(A, B$, and $D)$ in the intersection of the plane and the orthant. We leave the proof of this as an exercise for the reader.

Step 4. The conjecture of the fundamental domain was produced in the statement of this theorem.

Step 5. It remains to test the conjectured fundamental domain. For the test we need some extra points:

$$
\begin{aligned}
& E=X_{a, b}^{-1}(B)=(1,-a b-a-2 b-2, \\
& \left.\quad a^{2} b^{2}+2 a^{2} b+4 a b^{2}+a^{2}+8 a b+4 b^{2}+5 a+7 b+5\right), \\
& F=Y_{a, b}(B)=(-a-2,1,0), \\
& H=X_{a, b}^{-1}(F)=\left(0,-b-1, a b^{2}+2 a b+2 b^{2}+a+4 b+3\right) .
\end{aligned}
$$

1. (Test of condition i). It can be shown in the usual way that the faces have common edge $B D$, and the edges intersect only at vertices. This implies that all adjacencies are correct, and that only one or two faces are adjacent to each edge. The closure of the boundary is a closed broken line $A B C D A$, homeomorphic to the circle.

2. (Test of condition ii). By direct calculation it follows that by the operator $X_{a, b}$ action the segment $A B$ is taken to the segment $D C$ (the point $A$ maps to the point $D$ and $B$ to $C$ ) and by the operator $Y_{a, b}$ action the segment $A D$ is taken to the segment $B C$ (the point $A$ maps to the point $B$ and $D$ to $C$ ). Obviously, no other points glue together. The Euler characteristic of the obtained surface equals $2-3+1$, i.e. zero, and the surface is orientable.

3. (Calculation of all integer distances from the origin to the two-dimensional planes containing faces.) Let us calculate the integer distances from the origin to the two-dimensional planes containing faces $A B D$ and $B D C$ by the formula of Proposition 4.6. The integer distance to the plane of $A B D$ equals

$$
\frac{1}{b+1} \cdot\left|\left(\begin{array}{ccc}
1 & 0 & b^{2}+2 b+1 \\
0 & 0 & b+1 \\
a+1 & 1 & 1
\end{array}\right)\right|=\frac{b+1}{b+1}=1 .
$$


The integer distance from the origin to the plane of $B D C$ equals

$$
\frac{1}{b+1} \cdot\left|\left(\begin{array}{ccc}
0 & b^{2}+2 b+1 & b-a-1 \\
0 & b+1 & 1 \\
1 & 1 & 0
\end{array}\right)\right|=\frac{a b+2 b+a+2}{b+1}=a+2 .
$$

4. (Test on nonexistence of integer points inside the pyramids with vertices at the origin and bases at the faces.) Since the integer distance from the origin to the plane containing $A B D$ equals one, the pyramid corresponding to $A B D$ does not contain integer points different from $O$ and the points of the face $A B D$.

The face $B D C$ is integer-linear equivalent to the face with vertices $(1, a+1$, $-a-2),(b+2, a+1,-a-2),(1, a+2,-a-2)$ of the list of Theorem 4.9. The corresponding transformation taking $B C D$ to the face of the list of Theorem 4.9 is the following:

$$
\left(\begin{array}{ccc}
b+1 & b-a-1 & b-a \\
1 & 1 & 1 \\
0 & -1 & -1
\end{array}\right)
$$

By Theorem 4.9 the pyramid corresponding to $B D C$ does not contain integer points different from $O$ and the points of the face $B D C$.

5. (Test on convexity of dihedral angles.) Let us first consider the edge $B D$. This edge is adjacent to the faces $A B D$ and $B D C$. The face $A B D$ is contained in the plane $f_{A B D}(x, y, z)=0$, and the face $B D C$ is contained in the plane $f_{B D C}(x, y, z)=0$, where

$$
\begin{aligned}
& f_{A B D}(x, y, z)=(-1-a) x+(a b+a+b+1) y+z-1, \\
& f_{B D C}(x, y, z)=x+(b+1) y-(a+2) z+(a+2) .
\end{aligned}
$$

To test that the dihedral angle corresponding to the edge $B D$ is well placed it is sufficient to verify the following: the point $C$ and the origin $O$ lie in different halfspaces with respect to the plane spanned by the points $A, B$, and $D$; the points $A$ and $O$ lie in different half-spaces with respect to the plane spanned by the points $C, B$, and $D$. So we need to solve the following system:

$$
\left\{\begin{array}{l}
f_{A B D}(C) \cdot f_{A B D}(O)<0 \\
f_{B D C}(A) \cdot f_{B D C}(O)<0 .
\end{array}\right.
$$

This system is equivalent to the following one:

$$
\left\{\begin{aligned}
\left(a^{2}+3 a+2\right) \cdot(-1) & <0 \\
\left(-a^{2}-3 a-1\right) \cdot(a+2) & <0 .
\end{aligned}\right.
$$

Since $a \geq 0$, the inequalities hold. Thus the dihedral angle associated with the edge $B D$ is well placed. Since the cases of dihedral angles associated to the edges $A B$ (and the faces $A D B$ and $A E B$ ) and $B C$ (and the faces $B D C$ and $C B F$ ) can be verified in the same way, we omit their descriptions. This concludes the test of condition 5.

6. (Verification that all 2-stars of the vertices are regular.) There is only one vertex in the torus decomposition. Any lift of this point to the universal covering $W$ is adjacent to six edges and six faces. Consider a vertex of the universal covering that maps to the point $B$. The corresponding 2-star maps to six edges $B C, B D$, $B A, B E, B H$, and $B F$ and to six faces $B C D, B D A, B A E, B E H, B H F$, and $B F C$, where

$$
H=X_{a, b}^{-1}(F)=\left(0,-b-1, a b^{2}+2 a b+2 b^{2}+a+4 b+3\right) .
$$


We will check that for any sufficiently small positive $\varepsilon$ a ray $l_{\varepsilon}$ with vertex at the origin and passing through the point $P_{\varepsilon}=(\varepsilon, 0,1)$ intersects exactly one of the faces of 2-stars.

First we will check that for any sufficiently small positive $\varepsilon$ the ray $l_{\varepsilon}$ intersects the triangle $B C F$. Or, equivalently, that the ray $l_{\varepsilon}$ is contained in the trihedral angle with vertex at the origin $O$ and base in the triangle $B C F$. The two-dimensional face of the trihedral angle containing $B, C$, and $O$ can be defined by $f_{A B O}=0$; the two-dimensional face of the trihedral angle containing $B, F$, and $O$ can be defined by $f_{B F O}=0$; the two-dimensional face of the trihedral angle containing $C, F$, and $O$ can be defined by $f_{C F O}=0$, where

$$
\begin{aligned}
& f_{B C O}(x, y, z)=x+(a+1-b) y \\
& f_{B F O}(x, y, z)=x+(a+2) y, \\
& f_{C F O}(x, y, z)=z
\end{aligned}
$$

For any sufficiently small positive $\varepsilon$ the ray $l_{\varepsilon}$ is contained in the dihedral angle defined above if the following conditions hold: the points $P_{\varepsilon}$ and $F$ are in the same closed half-space with respect to the plane $f_{B C O}=0$; the points $P_{\varepsilon}$ and $C$ are in the same closed half-space with respect to the plane $f_{B F O}=0$; the points $P_{\varepsilon}$ and $B$ are in the same closed half-space with respect to the plane $f_{C F O}=0$. Since the points $P_{\varepsilon}$ and $B$ are close to each other for sufficiently small $\varepsilon$, they are in the same closed half-space with respect to the plane $f_{C F O}=0$. We now check the remaining two conditions:

$$
\left\{\begin{array} { l } 
{ f _ { B C O } ( P _ { \varepsilon } ) \cdot f _ { B C O } ( F ) \geq 0 , } \\
{ f _ { B F O } ( P _ { \varepsilon } ) \cdot f _ { B F O } ( C ) \geq 0 , }
\end{array} \Leftrightarrow \left\{\begin{array}{r}
(-b-1) \varepsilon \geq 0 \\
(b+1) \varepsilon \geq 0 .
\end{array}\right.\right.
$$

Since $b, \varepsilon \geq 0$ the first inequality does not hold. Thus for any sufficiently small positive $\varepsilon$ the ray $l_{\varepsilon}$ does not intersect the triangle $B C F$.

The cases of the triangles $B C D, B D A, B A E, B E H$, and $B H F$ are similar to those described above and are omitted here.

The ray $l_{\varepsilon}$ (for any sufficiently small positive $\varepsilon$ ) intersects the bijective image of a 2 -star of the vertex at exactly one point contained in the edge $A B$. Therefore, all 2-stars associated to the vertices are regular.

7. (Test that all the vertices of $D$ are in the same orthant.) The test of the seventh stage for this theorem is trivial since $D$ contains exactly one vertex.

\section{ACKNOWLEDGEMENTS}

The author is grateful to V. I. Arnold, E. I. Korkina, G. Lachaud, M. A. Tsfasman, and A. B. Sossinsky for useful remarks and discussions, and to the Institut de Mathématiques de Luminy (CNRS) for its hospitality and excellent working conditions.

\section{REFERENCES}

[1] V. I. Arnold, Continued fractions, Moscow: Moscow Center of Continuous Mathematical Education, (2002).

[2] V. I. Arnold, Higher-dimensional continued fractions, Regular and Chaotic Dynamics, 3(3), pp. 10-17, (1998). MR1704965 (2000h:11012)

[3] Z. I. Borevich, I. R. Shafarevich, Number theory, third ed., Moscow, (1985). MR0816135 (88f:11001)

[4] K. Briggs, Klein polyhedra, http://www.btexact.com/people/briggsk2/klein-polyhedra.html, (2002). 
[5] A. D. Bryuno, V. I. Parusnikov, Klein polyhedrals for two cubic Davenport forms, Mathematical notes, 56(4), (1994), pp. 9-27. MR.1330372 (96a:11061)

[6] H. Cohen, A Course in Computational Algebraic Number Theory, Graduate Texts in Mathematics, 138. Berlin, Springer-Verlag, (1973). MR1228206 (94i:11105)

[7] O. N. German, Sails and Hilbert Bases, Proc. of Steklov Ins. Math, 239, (2002), pp. 88-95. MR:1975137 (2004d:52017)

[8] O. N. German, Sails and Norm Minima of Lattices, Sb. Math., 196, (2005), pp. 337-365. MR2144275 (2006a:11087)

[9] C. Hermite, Letter to C. D. J. Jacobi, J. Reine Angew. Math. 40, (1839), p. 286.

[10] A. Ya. Hinchin, Continued fractions, Moscow: FISMATGIS, (1961).

[11] O. Karpenkov, On the triangulations of tori associated with two-dimensional continued fractions of cubic irrationalities, Funct. Anal. Appl., 38(2), (2004), pp. 102-110. MR2086625 (2005h:11150)

[12] O. N. Karpenkov, On two-dimensional continued fractions for integer hyperbolic matrices with small norm, Uspehi Mat. Nauk, 59(5), (2004), pp. 149-150. MR2125934 (2005m:11132)

[13] O. N. Karpenkov, On examples of two-dimensional periodic continued fractions, preprint, Cahiers du Ceremade, UMR 7534, Université Paris-Dauphine, (2004).

[14] O. N. Karpenkov, Classification of three-dimensional multistoried completely hollow convex marked pyramids, Uspekhi Mat. Nauk, 60(1), (2005), pp. 169-170. MR2145668 (2005m:11127)

[15] F. Klein, Uber eine geometrische Auffassung der gewöhnlichen Kettenbruchentwicklung, Nachr. Ges. Wiss. Göttingen Math-Phys. Kl., 3, (1895), pp. 357-359.

[16] F. Klein, Sur une représentation géométrique de développement en fraction continue ordinaire, Nouv. Ann. Math. 15(3), (1896), pp. 327-331.

[17] M. L. Kontsevich and Yu. M. Suhov, Statistics of Klein Polyhedra and Multidimensional Continued Fractions, Amer. Math. Soc. Transl., 197(2), (1999) pp. 9-27. MR.1733869 (2001h:11101)

[18] E. I. Korkina, The simplest 2-dimensional continued fraction, International Geometrical Colloquium, Moscow 1993

[19] E. I. Korkina, La périodicité des fractions continues multidimensionelles, C. R. Ac. Sci. Paris, 319, (1994), pp. 777-780. MR1300940 (95j:11064)

[20] E. I. Korkina, Two-dimensional continued fractions. The simplest examples, Proceedings of V. A. Steklov Math. Ins., 209, (1995), pp. 143-166. MR.1422222 (97k:11104)

[21] E. I. Korkina, The simplest 2-dimensional continued fraction., J. Math. Sci., 82(5), (1996), pp. 3680-3685. MR1428725 (97j:11032)

[22] G. Lachaud, Polyèdre d'Arnold et voile d'un cône simplicial: analogues du thèoreme de Lagrange, C. R. Ac. Sci. Paris, 317, (1993), pp. 711-716. MR1244417 (94m:11081)

[23] G. Lachaud, Voiles et Polyèdres de Klein, preprint n 95-22, Laboratoire de Mathématiques Discrètes du C.N.R.S., Luminy (1995).

[24] G. Lachaud, Sails and Klein Polyhedra, Contemp. Math., 210, (1998), pp. 373-385. MR.1478504 (98k:11094)

[25] A. K. Lenstra, H. W. Lenstra, Jr., and L. Lovász, Factoring Polynomials with Rational Coefficients, Mathematische Ann., 216(1982) pp. 515-534. MR682664 (84a:12002)

[26] H. Minkowski, Généralisation de le théorie des fractions continues, Ann. Sci. Ec. Norm. Super. ser III, 13, (1896), pp. 41-60. MR.1508923

[27] J.-O. Moussafir, Sails and Hilbert bases, Func. An. and Appl., 34(2), (2000), pp. 114-118. MR1773843 (2001d:52007)

[28] J.-O. Moussafir, Voiles et Polyédres de Klein: Geometrie, Algorithmes et Statistiques, docteur en sciences thése, Université Paris IX - Dauphine, (2000), see also http://www.ceremade.dauphine.fr/ ${ }^{\sim m s f r / ~}$

[29] R. Okazaki, On an effective determination of a Shintani's decomposition of the cone $\mathbb{R}_{+}^{n}$, J. Math. Kyoto Univ., 33(4), (1993), pp. 1057-1070. MR1251215 (94j:11117)

[30] V.I. Parusnikov, Klein's polyhedra for the third extremal ternary cubic form, preprint 137 of Keldysh Institute of the RAS, Moscow, (1995).

[31] V.I. Parusnikov, Klein's polyhedra for the fifth extremal cubic form, preprint 69 of Keldysh Institute of the RAS, Moscow, (1998).

[32] V.I. Parusnikov, Klein's polyhedra for the seventh extremal cubic form, preprint 79 of Keldysh Institute of the RAS, Moscow, (1999). 
[33] V.I. Parusnikov, Klein's polyhedra for the fourth extremal cubic form, Mat. Zametki, 67(1), (2000), pp. 110-128. MR1763552 (2001c:11072)

[34] T. Shintani, On evaluation of zeta functions of totally real algebraic number fields at nonpositive integers, J. Fac. Sci. Univ. Tokyo Sect. IA, 23, (1976), pp. 393-417. MR0427231 $(55: 266)$

[35] B. F. Skubenko, Minima of a decomposible cubic form of three variables, Sci. Seminar Notes LOMI, 168, (1988), Analytic Number Theory and Theory of Functions, 9, Leningrad, "Nauka".

[36] B. F. Skubenko, Minima of decomposible forms of degree $n$ of $n$ variables for $n \geq 3$, Sci. Seminar Notes LOMI, 183, (1990), Modular functions and quadratic forms, 1, Leningrad, "Nauka".

[37] E. Thomas and A. T. Vasques, On the resolution of cusp singularities and the Shintani decomposition in totally real cubic number fields, Math. Ann., 247, (1980), pp. 1-20. MR565136 (81h:10037)

[38] H. Tsuchihashi, Higher-dimensional analogues of periodic continued fractions and cusp singularities, Tohoku Math. Journ. 35, (1983), pp. 176-193. MR721966 (86a:14001)

[39] G. F. Voronoi, On a generalization of continued fraction algorithm, USSR Ac. Sci., 1, (1952), pp. 197-391.

Poncelet Laboratory (UMI 2615 of CNRS and Independent University of Moscow)

E-mail address: karpenk@mccme.ru 